\title{
Respiratory syncytial virus infection in high risk infants and the potential impact of prophylaxis in a United Kingdom cohort
}

\author{
Simon J Clark, Michael W Beresford, N V Subhedar, N J Shaw
}

\begin{abstract}
Background-Bronchiolitis caused by respiratory syncytial virus (RSV) is an important cause of morbidity in expremature infants. In a randomised placebo controlled trial monoclonal antibody prophylaxis showed a $55 \%$ reduction in relative risk of hospital admission for these high risk infants, against a background incidence of $\mathbf{1 0 . 6}$ admissions per 100 high risk infants.

Aims-To follow a cohort of high risk infants in order to assess hospitalisation rate from RSV and the potential impact of prophylaxis for these patients in a UK local health authority.
\end{abstract}

Methods-A cohort of high risk infants from a local health authority were followed over the $1998 / 99$ and $1999 / 2000 \mathrm{RSV}$ seasons. The high risk population was defined as infants who, at the beginning of the seasons studied, were: (1) under 6 months old and born prior to 36 weeks gestation with no domiciliary oxygen requirement; or (2) under 24 months of age and discharged home in supplemental oxygen. All admissions with bronchiolitis during the season were identified.

Results-A total of 370 high risk infants were identified for the $1998 / 99$ season and 286 for the following year. Over the two years there were 68 admissions. Significantly more admissions occurred from group 2 infants. RSV was identified in 27 cases (four admissions per hundred high risk infants). Prophylaxis may have saved up to $£ 195134$ in hospital costs over the two years, but would have cost $£ 1.1$ million in drug acquisition costs.

Conclusions-Careful consideration of risk factors is needed when selecting infants for RSV prophylaxis.

(Arch Dis Child 2000;83:313-316)

Keywords: respiratory syncytial virus; bronchiolitis; epidemiology; neonatal infections; prematurity; chronic lung disease

Survivors of premature delivery and recipients of neonatal intensive care, especially those with chronic lung disease (CLD), are at risk of increased morbidity and hospitalisation from bronchiolitis. ${ }^{1-4} \mathrm{Up}$ to $50 \%$ of these admissions may be caused by respiratory syncytial virus (RSV) infection. ${ }^{5}$ However, most of these data come from the control arms of large trials, in mainly North American populations. ${ }^{6}$ More recently the IMpact trial, a large randomised placebo controlled trial of intramuscular humanised monoclonal antibody prophylaxis against RSV, documented an admission rate with RSV of 10.6 per hundred high risk infants in the control group compared with the 4.8 per hundred high risk infants in the treatment arm. ${ }^{5}$ This represented a relative risk reduction of $55 \%$, but an absolute risk reduction of $5.8 \%$. The most pronounced effect of prophylaxis was seen in the ex-premature infants without CLD, in whom the relative risk reduction was $78 \%{ }^{5}$ Although prophylaxis significantly reduced the intensive care unit (ICU) admission rate, no statistically significant differences in the incidence of death, mechanical ventilation, or duration of individual hospital admission were seen. ${ }^{5}$ This form of RSV prophylaxis has been recently launched in the UK.

There is a paucity of accurate follow up data on UK high risk infants, making the potential impact of this new RSV prophylaxis difficult to assess. ${ }^{7}$ Joffe and colleagues have recently published a population based retrospective cohort follow up study based in northern California. ${ }^{6}$ They showed that proven RSV infection caused 3.5 admissions per hundred high risk infants. ${ }^{6}$ Unfortunately, $26.5 \%$ of infants were lost to follow up. ${ }^{6}$ These findings may not be applicable to the UK, especially since the Joffe cohort had under representation of lower socioeconomic groups. ${ }^{6}$

The aims of this prospective study were twofold. Firstly, to determine the hospital admission rate with RSV infection for a high risk cohort from a local health authority (LHA) population in the north of England. Secondly, to assess the potential impact that RSV prophylaxis would have had for these patients and the LHA over the 1998/99 and 1999/2000 RSV seasons.

\section{Methods}

The characteristics of the population studied in the IMpact trial were matched as closely as possible. ${ }^{5}$ The high risk population for this study was defined as infants who, at the beginning of the 1998/99 and 1999/2000 RSV seasons were: (1) under 6 months old and born prior to 36 weeks gestation with no requirement for domiciliary oxygen, including those discharged home during the RSV season; or (2) under 24 months of age and initially discharged home in supplemental oxygen, again including those discharged home during the RSV season. All infants who were born and were resident at home within the Liverpool LHA (total popula- 
Table 1 Cohort characteristics for the two seasons studied

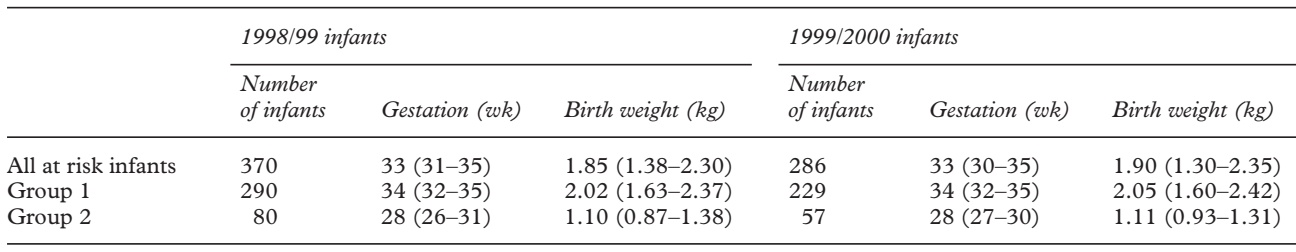

$\star$ Values shown are number or median (interquartile range).

Table 2 Number of admissions for each year and from the two subgroups

\begin{tabular}{|c|c|c|c|c|c|c|}
\hline & \multicolumn{2}{|c|}{ 1998/99 infants } & \multicolumn{2}{|c|}{ 1999/2000 infants } & \multicolumn{2}{|c|}{$1998 / 2000$} \\
\hline & Number & Admissions & Number & Admissions & Number & Admissions \\
\hline All at risk infants & 370 & $36(9.7 \%)$ & 286 & $32(11.2 \%)$ & 656 & $68(10.4 \%)$ \\
\hline Group 1 & 290 & $21(6.9 \%)$ & 229 & $14(6.1 \%)$ & 519 & $35(6.7 \%)$ \\
\hline Group 2 & 80 & $15^{\star}(18.8 \%)$ & 57 & $18^{\star}(31.6 \%)$ & 137 & $33^{\star}(24.1 \%)$ \\
\hline
\end{tabular}

${ }^{\star} \mathrm{p}<0.005$ compared with group 1 .

tion 492 100) during the study period were eligible for inclusion. Group 1 infants were born at either Liverpool Women's Hospital or Fazakerley Hospital, Aintree and were identified from the birth record databases. All infants from group 2 were identified from their attendance at a specialised clinic at the regional referral centre, Royal Liverpool Children's Hospital NHS Trust, Alder Hey. Residence within the Liverpool LHA was determined from the maternal postcode. The local RSV season studied was from 1 October to 31 March inclusive in 1998/99 and 1999/2000.

All infants from this high risk cohort who were subsequently admitted with a respiratory diagnosis to Alder Hey, which serves the entire population of the LHA and beyond, were identified from the admission database. RSV status was assessed for all admissions from the hospital's microbiological reports.

The average cost for each LHA infant admitted as an emergency and the average cost of a single intensive care day were obtained from the finance department at Alder Hey. Drug costs and dosage of humanised monoclonal antibody to RSV were obtained from the prescribing information sheet. ${ }^{8}$

Local research ethics committee approval was granted. Statistical analysis used the Mann-Whitney U test and $\chi^{2}$ test with Fisher's exact correction, where appropriate, within SPSS version 8.0 for Windows.

\section{Results}

The cohort of high risk infants, who met the inclusion criteria, for 1998/99 numbered 370 (75 high risk infants per 100000 population). The cohort for $1999 / 2000$ season numbered 286 (58 high risk infants per 100000 population). Group 1 comprised 290 and 229 infants for $1998 / 99$ and $1999 / 2000$ respectively.
Group 2 consisted of 80 infants for 1998/99 and 57 infants for 1999/2000. There were no significant differences between the gestation or birth weight for the infants between the two years (table 1).

Twenty eight infants were admitted with a respiratory illness consistent with bronchiolitis in the 1998/99 RSV season on 36 occasions; during the 1999/2000 RSV season 25 infants were admitted on 32 occasions. Over the two years this is equivalent to 10.4 admissions per 100 at risk infants, or 13.8 admissions per 100000 of LHA population. Table 2 shows the admissions from the subgroups over the two years. There were significantly more admissions in both years from group 2 than from group 1 . The odds ratio $(95 \%$ confidence interval) across the two years was $4.4(2.5-7.6)$ for admission from group 2 infants compared with group 1 . There were significantly more repeated admissions from group 2 than from group 1 over the two years. Over the two years, 32 group 1 infants were admitted, of whom two had repeated admissions; there were 21 group 2 infants admitted, eight of whom had more than one admission. The odds ratio (95\% confidence interval) for repeated admissions over the two years was $9.2(1.5-96.3)$ for group 2 $(8 / 21$ or $38 \%)$ compared with group 1 ( $2 / 32$ or $6 \%$ ). The odds ratio ( $95 \%$ confidence interval) for the number of infants admitted, therefore not counting repeated admissions, over the two years was $2.8(1.5-5.1)$ for group $2(21 / 137$ or $15 \%)$ compared with group 1 (32/519 or $6 \%)$. Table 3 shows the numbers of infants admitted, age at admission, length of stay, and intensive care days. Five infants were admitted to the intensive care unit (ICU) over the two years for between 1 and 86 days. Two were group 1 and three were group 2 infants. Only one group 2 infant died, who contracted RSV while in hos-

Table 3 Number of infants admitted, age at admission, and length of stay, including ICU admissions

\begin{tabular}{|c|c|c|c|c|c|c|c|c|}
\hline & $\begin{array}{l}\text { Number of } \\
\text { infants admitted }\end{array}$ & $\begin{array}{l}\text { Admission } \\
\text { age (mth) }\end{array}$ & $\begin{array}{l}\text { Length of } \\
\text { stay (days) }\end{array}$ & $\begin{array}{l}\text { Total hospital } \\
\text { days }\end{array}$ & $\begin{array}{l}\text { ICU } \\
\text { admissions }\end{array}$ & $\begin{array}{l}\text { Length of ICU } \\
\text { stay (days) }\end{array}$ & $\begin{array}{l}\text { Total ICU } \\
\text { days }\end{array}$ & $\begin{array}{l}\text { ICU admissions } \\
\text { age (mth) }\end{array}$ \\
\hline All at risk infants $(n=656)$ & $53(8.1 \%)$ & $5(3-10)$ & $4(2-7)$ & 423 & $5(9.4 \%)$ & $4(2-7)$ & 102 & $2 \quad(2-3)$ \\
\hline Group $1(n=519)$ & $32(6.2 \%)$ & $4(2-5)$ & $3(2-6)$ & 155 & $2(6.3 \%)$ & $7(5-10)$ & 14 & $1.5(1-2)$ \\
\hline Group $2(\mathrm{n}=137)$ & $21^{\star}(15.3 \%)$ & $11(4-14)$ & $4(2-8)$ & 268 & $3(14.3 \%)$ & $1(1-44)$ & 88 & $3(3-4)$ \\
\hline
\end{tabular}

Values shown are number (percentage) or median (interquartile range).

${ }^{\star} \mathrm{p}=0.001$ compared with group 1 . 
Table 4 Minimum cost and maximum saving available to the LHA over 1998/2000

\begin{tabular}{llll}
\hline & Maximum savings & Minimum cost & $\begin{array}{l}\text { Minimal additional } \\
\text { cost to the LHA }\end{array}$ \\
\hline All at risk infants & $£ 95134$ & $£ 1160799^{\star}$ & $£ 965665$ \\
Group 1 & $£ 48650$ & $£ 883831^{\star}$ & $£ 835181$ \\
Group 2 & $£ 146484$ & $£ 461177 \dagger$ & $£ 314693$ \\
\hline
\end{tabular}

^Assumes infants weigh less than $3.3 \mathrm{~kg}$ at the time of immunisation.

†Assumes group 2 infants weigh between 3.3 and $6.6 \mathrm{~kg}$ at the time of immunisation.

pital; however, RSV did not appear on the death certificate.

RSV testing was not universally performed in all admissions. RSV status was identified in only 33 admissions. Twenty seven of these were positive and six were negative. Therefore, based on this cohort, proven RSV caused 4.1 admissions per hundred high risk infants during the seasons studied. It is possible, although unlikely, that up to 62 of the 68 admissions over the two years were secondary to RSV.

The calculation of hospital costs has used two admission rates. One assumes that only the 27 documented cases were caused by RSV and that the other 62 were RSV related. Therefore, from the IMpact trial data a $55 \%$ reduction in admissions from RSV prophylaxis might be expected to have averted a range of 15 to 34 admissions, saving £16 500-37 400. If all ICU admissions had been avoided a further saving of $£ 157734$ may have been realised. The potential range of savings to the LHA could have ranged from $£ 16500$ to $£ 195134$ over the two years.

If the whole cohort of high risk infants had been given monoclonal antibody prophylaxis, the total number of injections required would have been 2738 over the two years. The dose is weight dependent, therefore making cost weight dependent; assuming that all infants weighed less than $3.3 \mathrm{~kg}$ at the time of injection provides the lowest estimate of cost at $£ 1$.1 million. It is more likely that the infants weighed between 3.3 and $6.6 \mathrm{~kg}$ at the time of injection and this would have cost $£ 1.9$ million. Table 4 shows the maximum savings possible and the minimum costs for the cohort and subgroups.

\section{Discussion}

In an LHA with a total population of 492100 we have found 656 infants at high risk from RSV infection. An admission rate of 10.4 per hundred high risk infants with bronchiolitis was seen across the two years. Only 4.1 admissions per hundred high risk infants were proven RSV infection. This is considerably lower than the 10.6 per hundred high risk infants found in the placebo arm by the IMpact Study group, ${ }^{5}$ and provides important UK epidemiological data. Interestingly, this is very similar to the admission rate of 3.2 per hundred high risk infants reported by Joffe and colleagues. ${ }^{6}$ It is possible that the 35 infants in which no record of RSV testing could be found were RSV positive. This, however, would mean that all but six cases of bronchiolitis in this high risk cohort were caused by RSV, which is unlikely.

The diagnosis of bronchiolitis for our study was taken from the admissions database at the local paediatric hospital. This is the data set used to complete their annual returns and therefore represents an accurate reflection of activity, although it is possible that miscoding may have led to some infants being missed. The infants enrolled in the IMpact trial were closely followed up. RSV testing was performed on all infants in the study if admitted to hospital for any reason. The admission was deemed to be a respiratory admission with RSV if the child was hospitalised for a respiratory illness and the RSV test was positive or if the child had a lower respiratory illness/ infection score 3 or more ${ }^{5}$ during any admission and the RSV test was positive. It is possible that this may have led to over reporting of the RSV rate as infants could be hospitalised with RSV rather than because of RSV.

A geographically based area was chosen to define this study's cohort, enabling the maximal follow up of infants as only one paediatric hospital serves the entire area studied. The only infants excluded would have been those, while outside the region, who were born prematurely or admitted to hospital with RSV. We used two consecutive years to reduce the effect that the year on year variation in bronchiolitis rates would have on our assessment. While acknowledging that the incidence of RSV may vary in different years and that levels of deprivation may affect the local RSV incidence, these data give paediatricians an assessment of the risk of RSV infection in a UK LHA.

The high risk population was matched as closely as possible to that of the IMpact trial. ${ }^{5}$ However, their group 2 infants were enrolled if they had had bronchopulmonary dysplasia (an oxygen requirement at 28 days of age) requiring ongoing medical treatment within the last six months. Using this definition would have made it much more difficult to follow up these infants in this study, as any mildly wheezy infant receiving bronchodilator therapy in the community would have been eligible for RSV prophylaxis. This milder degree of underlying illness is probably why this subgroup showed less treatment advantage in the IMpact trial with only a $39 \%$ reduction in hospitalisations. Our subpopulation would be anticipated, if anything, to show greater potential benefit from this intervention. ${ }^{34}$

The actual cost for an individual admission to hospital is difficult to quantify. The average cost of an admission is usually taken as the total cost of the service divided by the number of admissions, weighted for length of stay. This represents the full cost of the admission including infrastructure and staffing. Therefore, this cannot be taken as the savings that would be released from the decision not to admit one patient. Although up to 34 admissions may have been potentially avoided in this study, it is unlikely that this would have prevented the opening of a winter pressure ward. Therefore, in reality the actual savings from the potentially reduced activity may be insignificant compared to the total treatment cost. We have made no attempt to estimate concrete and psychosocial costs to the infant 
and family that avoiding hospital admission may bring.

The overall $55 \%$ reduction in hospital admissions in the IMpact trial is clinically significant as well as statistically so. The prophylaxis required repeated intramuscular injections; despite this route of administration, the IMpact trial had a $99 \%$ compliance rate in both active and placebo arms of the trial. ${ }^{5}$ The high risk infants identified in the LHA in this study would have required 2738 intramuscular injections, an average of four per infant. Compliance with the treatment regimen may not be as satisfactory outside the trial setting.

The estimate of drug acquisition costs was calculated as accurately as possible. This expense may be an overestimate, as discounting may be available for hospital purchasing. However, if the drug were administered mainly in a primary care setting the additional expense of organising immunisation clinics and staffing costs would also need to be taken into account.

We attempted to stratify our infants in terms of risk, group 2 being those who were most likely to be admitted to hospital and the most likely to have repeated admissions. Despite this the difference in cost of drug acquisition and the savings released to the health authority was still over $£ 300000$ in the group 2 infants. This shortfall may be a conservative estimate as in the group 2 infants in the IMpact trial there was less of an effect of immunisation. ${ }^{5}$

This study has shown a lower than expected RSV infection rate in high risk infants compared with previous trial data. There was no significant variation between the admission rates for the two years. It would seem that a particularly high risk subgroup of infants needs to be identified so that RSV prophylaxis can be targeted by paediatricians and LHAs to where potential outcome benefits outweigh the costs involved. Although we have shown our group 2 infants to be at greater risk of admission and repeated admissions, there may be additional factors such as weight at discharge from the neonatal unit, number of siblings, and perhaps even parental smoking habits that need to be taken into account, when deciding who would benefit most from RSV prophylaxis.

We wish to acknowledge Elaine Morgan and Steve Warburton at the Royal Liverpool Children's Hospital, Alder Hey; Keith Jones and Helen Jones at Aintree NHS Hospital Trust, Fazakerly; and Bill Yoxall at Liverpool Women's Hospital NHS Trust, Liverpool; and Jan Nolan at Liverpool Women's Hospital, Liverpool.

1 Meert K, Heidemann S, Abella B, Sarnaik A. Does prematurity alter the course of respiratory syncytial virus infection? Crit Care Med 1990;18:1357-9.

2 Kristensen K, Dahm T, Frederiksen PS, et al. Epidemiology of respiratory syncytial virus infection requiring hospitali-
sation in East Denmark. Pediatr Infect Dis $\mathcal{f} 1998 ; 17: 996-$ 1000 .

3 Groothuis JR, Gutierrez KM, Lauer BA. Respiratory syncytial virus infection in children with bronchopulmonary dysplasia. Pediatrics 1988;82:199-203.

4 Meert K, Heidemann S, Lieh-Lai M, Sarnaik A. Clinical characteristics of respiratory syncytial virus infections in healthy versus previously compromised host. Pediatr Pulmonol 1989;7:167-70.

5 The Impact-RSV Study Group. Palivizumab, a humanised respiratory syncytial virus monoclonal antibody, reduces hospitalisation from respiratory syncytial virus infection in high-risk infants. Pediatrics 1998;102:531-7.

6 Joffe S, Ray GT, Escobar GJ, Black SB, Lieu TA. Cost-effectiveness of respiratory syncytial virus prophylaxis among preterm infants. Pediatrics 1999;104:419-27.

7 Joffe S, Escobar GJ, Black SB, Armstrong MA, Lieu TA. Rehospitalization for respiratory syncytial virus among preterm infants. Pediatrics 1999;104:894-9.

8 Anon. Synagis (palivizumab) launch information for NHS Anon. Synagis (palivizumab) launch information for NHS
purchasers. Abbott Laboratories Limited. September 1999 .

\section{Invitation to Delegates}

\section{6th European Forum on Quality Improvement in Health Care Thursday 29-Saturday 31 March 2001, Bologna, Italy}

For full information, contact BMA/BMJ Conference Unit, BMA House, Tavistock Square, London WC1H 9JP UK. Tel +44 (0) 207383 6409; fax: +44 (0) 207383 6869; email: quality@bma.org.uk

Bookmark our website for full information: www.quality.bmjpg.com 